\title{
ON COMBINING CORRELATED ESTIMATORS OF THE COMMON MEAN OF A MULTIVARIATE NORMAL DISTRIBUTION
}

\author{
K. KRISHNAMOORTHY ${ }^{1}$ and YONG LU \\ Department of Mathematics, University of Louisiana at Lafayette \\ Lafayette, LA 70504-1010, USA
}

The inferential procedures based on an optimal combination of correlated estimators of the common mean of a multivariate normal distribution are considered. Exact properties of the conditional and unconditional confidence intervals due to Halperin (1961) are numerically evaluated. Our numerical studies show that the conditional confidence interval is slightly shorter than the unconditional confidence interval. A condition under which the conditional approach is advantageous over the best of the $t$ procedures based on individual components is discussed. The methods are illustrated using an example.

Key words: Concomitant variable; expected length; maximum likelihood estimator; noncentral $t$ distribution; multiple correlation coefficient; power

${ }^{1}$ Corresponding Author:

e-mail: krishna@louisiana.edu

Phone: 337-482-5382; Fax: 337-482-5346 


\section{INTRODUCTION}

The problem of combining independent estimators for the common mean of several normal populations is well-known and has been well addressed in the literature. An important result in the common mean problem is due to Graybill and Deal (1956) who first showed, for the two-sample case, that the weighted average of the sample means with weights inversely proportional to their variances has smaller variance than either sample mean provided the sample sizes are greater than 10. Since then many authors improved and extended this result to the case of more than two populations, and developed several methods for hypothesis testing and interval estimation for the common mean. For a good exposition of the work in this area, we refer to Cohen and Sckrowitz (1984), Zhou and Mathew (1993), Yu, Sun and Sinha (1999) and Krishnamoorthy and $\mathrm{Lu}$ (2003) and the references therein. However, the results on combining the correlated estimators in the normal case are very limited. Halperin (1961) seems to be the first paper addressed this problem. Halperin pointed out that the problem of estimating the common mean of a multivariate normal population arises when several alike neutron transportation problems are considered. Halperin derived the maximum likelihood estimator (MLE) and developed two interval estimates for the common mean of a multivariate normal population.

We shall now describe the setup of the problem as given in Halperin (1961). Let $\mathbf{U} \sim N_{p}(\mathbf{e} \mu, \Sigma)$, where e denotes the vector of ones. Let $\overline{\mathbf{U}}$ and $S_{u}$ denote respectively the mean and covariance matrix based a sample of $n$ observations from $N_{p}(\mathbf{e} \mu, \Sigma)$. The maximum likelihood estimator of $\mu$ due to Halperin (1961) is given by

$$
\hat{\mu}=\frac{\mathbf{e}^{\prime} S_{u}^{-1} \overline{\mathbf{U}}}{\mathbf{e}^{\prime} S_{u}^{-1} \mathbf{e}}
$$

If $\Sigma$ is known, then the best linear unbiased estimator (BLUE) of $\mu$ is given by $\mathbf{e}^{\prime} \Sigma^{-1} \overline{\mathbf{U}} /\left(\mathbf{e}^{\prime} \Sigma^{-1} \mathbf{e}\right)$ and it has variance $\left(n \mathbf{e}^{\prime} \Sigma^{-1} \mathbf{e}\right)^{-1}$. If $\Sigma$ is unknown, replacing $\Sigma$ by its estimate $S_{u}$, we can get the MLE. The variance of the MLE is given by

$$
\operatorname{Var}(\hat{\mu})=\left(1+\frac{p-1}{n-p-1}\right) \frac{1}{n \mathbf{e}^{\prime} \Sigma^{-1} \mathbf{e}}
$$

which approaches the variance of the BLUE as $n \rightarrow \infty$.

The form of the MLE in (1) is not conducive to develop a confidence interval for $\mu$. To derive the distribution of the MLE, Halperin suggested to use the following transformation. Let $A=\left(a_{i j}\right)$ be a $p \times p$ matrix such that $a_{i 1}=1$ for $i=1, \ldots, p, a_{i i}=-1$ for $i=2, \ldots, p$, and $a_{i j}=0$ elsewhere. Then $A \mathbf{U}=\left(y, x_{1}, \ldots, x_{p-1}\right)^{\prime}=\left(y, X^{\prime}\right)^{\prime}$ follows a $p$-variate normal distribution with mean vector $(\mu, 0, \ldots, 0)^{\prime}$ and covariance matrix

$$
A \Sigma A^{\prime}=\left(\begin{array}{cc}
\sigma_{y y} & \sigma_{X y}^{\prime} \\
\sigma_{X y} & \Sigma_{X X}
\end{array}\right)_{p \times p} .
$$

Thus, estimation of the common mean $\mu$ is equivalent to estimation of the mean of $y$ given that the mean of $X=0_{p-1}$. Let $\left(y_{1}, X_{1}\right), \ldots,\left(y_{n}, X_{n}\right)$ be independent observations on $(y, X)$. Define

$$
\left(\bar{y}, \bar{X}^{\prime}\right)=\frac{1}{n} \sum_{i=1}^{n}\left(y_{i}, X_{i}^{\prime}\right)
$$


and

$$
\mathbf{W}_{p \times p}=\left(\begin{array}{cc}
w_{y y} & W_{X y}^{\prime} \\
W_{X y} & W_{X X}
\end{array}\right)=\left(\begin{array}{cc}
\sum_{i=1}^{n}\left(y_{i}-\bar{y}\right)^{2} & \sum_{i=1}^{n}\left(y_{i}-\bar{y}\right)\left(X_{i}-\bar{X}\right)^{\prime} \\
\sum_{i=1}^{n}\left(y_{i}-\bar{y}\right)\left(X_{i}-\bar{X}\right) & \sum_{i=1}^{n}\left(X_{i}-\bar{X}\right)\left(X_{i}-\bar{X}\right)^{\prime}
\end{array}\right),
$$

so that $W_{X X}$ is a $(p-1) \times(p-1)$ matrix. Let $\mathbf{a}=\left(a_{1}, \ldots, a_{p-1}\right)^{\prime}$ be a vector of real numbers, and $\beta=\Sigma_{X X}^{-1} \sigma_{X y}$. Consider the class of estimators of the form $\bar{y}(\mathbf{a})=\bar{y}-\sum_{i=1}^{p-1} a_{i} \bar{x}_{i}$. It can be easily shown that $\operatorname{Var}(\bar{y}(\mathbf{a}))$ is minimized when $\mathbf{a}=\beta$. Thus, if $\beta$ is known, then $\bar{y}(\beta)$ is the best linear unbiased estimator of $\mu$. If $\beta$ is unknown, then replacing it by $\mathbf{b}=W_{X X}^{-1} W_{X y}$ we get

$$
\hat{\mu}=\bar{y}-\mathbf{b}^{\prime} \bar{X}
$$

This is an alternative form of the MLE in (1). The expression for the variance of the MLE can be written as

$$
\operatorname{Var}(\hat{\mu})=\left(1+\frac{p-1}{n-p-1}\right) \frac{\sigma_{y y \cdot X}}{n}, \text { for } n>p+1,
$$

where $\sigma_{y y . X}=\sigma_{y y}\left(1-\rho_{y \cdot X}^{2}\right)$, and $\rho_{y \cdot X}^{2}=\left(\sigma_{X y}^{\prime} \Sigma_{X X}^{-1} \sigma_{X y}\right) / \sigma_{y y}$ is the squared multiple correlation coefficient between $y$ and $X$. It follows from (17) that the variance of the MLE is smaller than that of $\bar{y}=\bar{u}_{1}$ if and only if

$$
\rho_{y \cdot X}^{2}>\frac{p-1}{n-2} .
$$

Recall that the transformation we used is $\left(u_{1}, u_{1}-u_{2}, \ldots, u_{1}-u_{p}\right)=\left(y, x_{1}, \ldots, x_{p-1}\right)$. If we let $\left(u_{2}, u_{2}-u_{1}, \ldots, u_{2}-u_{p}\right)=\left(y, x_{1}, \ldots, x_{p-1}\right)$, then the MLE has smaller variance than $\bar{u}_{2}$ if and only if $\rho_{u_{1} .\left(u_{1}-u_{2}\right), \ldots,\left(u_{1}-u_{p}\right)}^{2}>(p-1) /(n-2)$. Proceeding this way, we see that the MLE has smaller variance than the $\min \left\{\operatorname{Var}\left(\bar{u}_{1}\right), \ldots, \operatorname{Var}\left(\bar{u}_{p}\right)\right\}$ if and only if

$$
\min \left\{\rho_{u_{1} .\left(u_{1}-u_{2}\right), \ldots,\left(u_{1}-u_{p}\right)}^{2}, \ldots, \rho_{u_{p} .\left(u_{p}-u_{1}\right), \ldots,\left(u_{p}-u_{p-1}\right)}^{2}\right\}>\frac{p-1}{n-2} .
$$

Krishnamoorthy and Rohatgi (1990) showed that $\hat{\mu}$ can be improved using the fact that the mean of $X$ is known to be zero. In particular, they suggested using $W_{X 0}=\sum_{i=1}^{n} X_{i} X_{i}^{\prime}$ to estimate $\beta$. This leads to the estimator $\hat{\mu}_{1}=\bar{y}-\mathbf{b}_{0}^{\prime} \bar{X}$, where $\mathbf{b}_{0}=W_{X 0} W_{X y}$ and $W_{X y}$ is defined in (5). Krishnamoorthy and Rohatgi (1990) showed that $\hat{\mu}_{1}$ has smaller variance than $\hat{\mu}$ over a wide range of parameter space.

The problem of estimating the mean of $y$ given that the mean of $X$ is $0_{p-1 \times 1}$ has been considered by Berry (1987), Tan and Gleser (1993), and Jin and Berry (1993). These authors refer to the vector $X$ as concomitant control vector for estimating the mean $\mu$ of $y$. This problem is equivalent to the common mean problem with the transformed variables. However, the main interest in the common mean problem is to develop a better inferential procedure, based on a combination of the correlated estimators, than the best of the $t$ procedures based on individual estimators whereas there is no such interest in the problem of estimating $\mu$ with a concomitant control vector.

In this article, we are mainly interested in comparing three confidence intervals, including the $t$-interval based on the marginal distribution of $y$, that are given in Halperin (1961). In the following section, we describe the conditional interval and the unconditional interval due 
to Halperin, and present expressions for their expected lengths. The expected lengths are compared numerically. Our comparison studies in Section 3 shows that the conditional intervals are either slightly shorter than or almost close to the unconditional intervals for all the cases considered. We also discuss a condition under which the expected length of the conditional confidence interval is shorter than the best of the $t$-intervals based on individual means. For the sake completeness, we also present the test based on the conditional approach, and its power function. The methods are illustrated using a simulated data set.

\section{INTERVAL ESTIMATION AND EXPECTED LENGTHS}

In the following lemma, we present some basic distributional results related to the statistics defined in the previous section. These results can be found, for example, in Muirhead (1982, Chapter 3).

\section{Lemma 2.1.}

(i) The conditional distribution of $\mathbf{b}=W_{X y}^{\prime} W_{X X}^{-1}$ given $\left(X_{1}, \ldots, X_{n}\right)$ is $N_{p-1}\left(\beta, \sigma_{y y \cdot X} W_{X X}^{-1}\right)$.

(ii) $n \bar{X} \Sigma_{X X}^{-1} \bar{X}=\left(\sqrt{n} \bar{X}^{\prime} \Sigma^{-\frac{1}{2}}\right)\left(\Sigma^{-\frac{1}{2}} \bar{X} \sqrt{n}\right)=Z^{\prime} Z \sim \chi_{p-1}^{2}$.

(iii) $V=\frac{\bar{X} \Sigma_{X X}^{-1} \bar{X}}{\bar{X} W_{X X}^{-1} \bar{X}} \sim \chi_{n-p+1}^{2}$ independently of $\bar{X}$ (or $Z$ )

(iv) $Q=n \bar{X}^{\prime} W_{X X}^{-1} \bar{X}=Z^{\prime} Z / V \sim \frac{p-1}{n-p+1} F_{p-1, n-p+1}$, where $F_{a, b}$ denotes the $F$ random variable with the numerator $\mathrm{df}=a$ and the denominator $\mathrm{df}=b$.

(vi) The sample conditional variance of $y$ given $X$ is defined as $\hat{\sigma}_{y y \cdot X}=\frac{w_{y y}-W_{X y}^{\prime} W_{X X}^{-1} W_{X y}}{n-p}$ and is distributed as $\frac{\sigma_{y y . X}}{n-p} \chi_{n-p}^{2}$ independently of $Q$.

We shall now present the confidence intervals that will be considered for comparison.

\subsection{The t-interval}

The usual $t$-interval based on the marginal distribution of $y$ is given by

$$
\bar{y} \pm t_{n-1,1-\alpha / 2} \sqrt{\frac{s_{y y}}{n}},
$$

where $s_{y y}$ is the sample variance of $y$ and $t_{m, \alpha}$ denotes the $\alpha$ th quantile of the Student's $t$ distribution. The expected length of the $t$-interval is given by

$$
E L_{1}=2 t_{n-1,1-\alpha / 2} E\left(\sqrt{\frac{s_{y y}}{n}}\right)=2 t_{n-1,1-\alpha / 2} \sqrt{\frac{2}{n}} \frac{\Gamma(n / 2)}{\Gamma(n-1) / 2} \sqrt{\frac{\sigma_{y y}}{n-1}},
$$

where $\Gamma($.$) denotes the gamma function.$ 


\subsection{The Conditional Interval}

We shall now describe the conditional confidence interval due to Halperin (1961). Using the results of Lemma 2.1, it can be readily verified that the conditional distribution of $\hat{\mu}$ given $X_{1}, \ldots, X_{n}$ is normal with mean $\mu$ and variance $\sigma_{y y . X}(1+Q)$, where $Q=n \bar{X}^{\prime} W_{X X}^{-1} \bar{X}$ defined in Lemma 2.1(v). We write

$$
\hat{\mu} \mid\left(X_{1}, \ldots, X_{n}\right) \sim N\left(\mu, \sigma_{y y . X}(1+Q) / n\right) .
$$

Notice that $(n-p) \hat{\sigma}_{y y . X} / \sigma_{y y . X} \sim \chi_{n-p}^{2}$. Using this result, we see that, conditionally given $Q$, the pivotal quantity

$$
\frac{\sqrt{n}(\hat{\mu}-\mu)}{\sqrt{\hat{\sigma}_{y y \cdot X}(1+Q)}} \sim t_{n-p} .
$$

This leads to the conditional confidence interval

$$
\hat{\mu} \pm t_{n-p, 1-\alpha / 2}(1+Q)^{\frac{1}{2}} \sqrt{\frac{\hat{\sigma}_{y y \cdot X}}{n}} .
$$

It follows from Lemma 2.1(iv) that $(1+Q)$ is distributed as $U^{-1}$, where $U$ is a beta random variable with parameters $(n-p+1) / 2$ and $(p-1) / 2$. Using this result and the fact that $\bar{X}^{\prime} W_{X X}^{-1} \bar{X}$ and $\hat{\sigma}_{y y . X}$ are independent, it is easy to see that the expected length of the conditional confidence interval in (13) is

$$
E L_{2}=2 t_{n-p, 1-\alpha / 2} \sqrt{\frac{2}{n}} \frac{\Gamma\left(\frac{n}{2}\right)}{\Gamma\left(\frac{n-1}{2}\right)}\left(\frac{\sigma_{y y}\left(1-\rho_{y \cdot X}^{2}\right)}{n-p}\right)^{\frac{1}{2}} .
$$

It should be noted that the formula for $E L_{2}$ given in Halperin (1961) is incorrect.

\subsection{The Unconditional Confidence Interval}

It follows from (12) and Lemma 2.1(iv) that

$$
T=\frac{\sqrt{n}\left(\bar{y}(\mathbf{b})-\mu_{0}\right)}{\sqrt{\hat{\sigma}_{y y . X}}} \sim t_{n-p}\left(1+\frac{p-1}{n-p+1} F_{p-1, n-p+1}\right)^{\frac{1}{2}} .
$$

The percentiles of $T$ can be used to form a $1-\alpha$ confidence interval for $\mu$. Using some standard methods, it can be shown that the $(1-\alpha)$ th quantile $k$ of $T$ is the solution of the equation

$$
\frac{\Gamma(n / 2)}{\Gamma((p-1) / 2) \Gamma((n-p+1) / 2)} \int_{0}^{1} G(k \sqrt{1-x} ; n-p) x^{(p-1) / 2-1}(1-x)^{(n-p+1) / 2-1} d x=1-\alpha,
$$

where $G(. ; m)$ denotes the Student's $t$ cdf with $\mathrm{df}=m$. To get $(16)$, we used the fact that $F_{a, b}$ is distributed as $b U /(a(1-U))$, where $U$ is a beta $(a / 2, b / 2)$ random variable. Noting that the Student's $t$ distribution is symmetric about zero, it follows from (16) that the distribution of $T$ is also symmetric about zero. Let $T_{\alpha}$ denote the $\alpha$ th quantile of $T$. Then, the unconditional $1-\alpha$ confidence interval for $\mu$ is given by

$$
\hat{\mu} \pm T_{1-\alpha / 2} \sqrt{\frac{\hat{\sigma}_{y y \cdot X}}{n}} .
$$


The expected length of the unconditional confidence interval is given by

$$
E L_{3}=2 T_{1-\alpha / 2} E\left(\sqrt{\frac{\hat{\sigma}_{y y \cdot X}}{n}}\right)=2 T_{1-\alpha / 2} \sqrt{\frac{2}{n}} \frac{\Gamma\left(\frac{n-p+1}{2}\right)}{\Gamma\left(\frac{n-p}{2}\right)}\left(\frac{\sigma_{y y}\left(1-\rho_{y \cdot X}^{2}\right)}{n-p}\right)^{\frac{1}{2}} .
$$

Remark 1. Using (16), we computed the values of $T_{1-\alpha / 2}$ when $\alpha=0.05$ and $0.1, p=2,3,4$ and 5, and values of $n$ ranging from 6 to 1000. These critical values are presented in Table I. We also found that the distribution of $T$ in (15) can be approximated by $c t_{n-p}$, where $c=$ $\sqrt{(n-2) /(n-p-1)}$. The constant $c$ was obtained by solving the equation $E\left(c^{2} t_{n-p}^{2}\right)=E\left(T^{2}\right)$. Using this approximation, we have $T_{1-\alpha / 2} \doteq t_{n-p, 1-\alpha / 2} \sqrt{(n-2) /(n-p-1)}$. This approximation is satisfactory as long as $n-p \geq 4$.

\section{COMPARISON OF EXPECTED LENGTHS}

It is clear from the expressions of $E L_{1}, E L_{2}$ and $E L_{3}$, that the ratios $E L_{2} / E L_{1}$ and $E L_{3} / E L_{1}$ depend on the parameter space only through $\rho_{y . X}^{2}$. Using this fact, direct comparison between $E L_{2}$ and $E L_{1}$ shows that the expected length of the conditional confidence interval is shorter than the expected length of the usual $t$ interval based on $y$ observations alone if and only if

$$
\rho_{y \cdot X}^{2}>1-\left(\frac{t_{n-1,1-\alpha / 2}}{t_{n-p, 1-\alpha / 2}}\right)^{2}\left(\frac{n-p}{n-1}\right) .
$$

The above inequality is different from the one given in Halperin (1961, p.41), because, as we already pointed out, Halperin's formula for the expected length of the conditional interval is incorrect. For fixed $p$, the right-hand side of (19) approaches zero as $n \rightarrow \infty$. This implies that, for large $n, E L_{2}$ is smaller than $E L_{1}$ for all practically meaningful values of $\rho_{y . X}^{2}$. However, this does not mean that $E L_{2}$ is smaller than the expected length of the shortest of the individual $t$-intervals. The above condition merely implies that $E L_{2}$ is smaller than the expected length of the $t$-interval based on $\bar{u}_{1}=\bar{y}$. For $E L_{2}$ to be shorter than the $t$-interval based on $\bar{u}_{2}$, we should have

$$
\rho_{u_{2} .\left(u_{2}-u_{1}\right), \ldots,\left(u_{2}-u_{p}\right)}^{2}>1-\left(\frac{t_{n-1,1-\alpha / 2}}{t_{n-p, 1-\alpha / 2}}\right)^{2}\left(\frac{n-p}{n-1}\right),
$$

where $\rho_{u_{2} .\left(u_{2}-u_{1}\right), \ldots,\left(u_{2}-u_{p}\right)}^{2}$ is the squared multiple correlation coefficient between $U_{2}$ and $\left(\left(U_{2}-\right.\right.$ $\left.\left.U_{1}\right), \ldots,\left(U_{2}-U_{p}\right)\right)$. Proceeding this way, we see that $E L_{2}$ is shorter than the shortest of the $t$-intervals if and only if

$$
\min \left\{\rho_{u_{1} .\left(u_{1}-u_{2}\right), \ldots,\left(u_{1}-u_{p}\right)}^{2}, \ldots, \rho_{u_{p} .\left(u_{p}-u_{1}\right), \ldots,\left(u_{p}-u_{p-1}\right)}^{2}\right\}>1-\left(\frac{t_{n-1,1-\alpha / 2}}{t_{n-p, 1-\alpha / 2}}\right)^{2}\left(\frac{n-p}{n-1}\right) .
$$

Comparison between $E L_{2}$ and $E L_{3}$ shows that the ratio $E L_{2} / E L_{3}>1$ if and only if

$$
t_{n-p, 1-\alpha / 2} \frac{\Gamma\left(\frac{n}{2}\right)}{\Gamma\left(\frac{n-1}{2}\right)}<T_{1-\alpha / 2} \frac{\Gamma\left(\frac{n-p+1}{2}\right)}{\Gamma\left(\frac{n-p}{2}\right)} .
$$


TABLE I Critical points $T_{1-\alpha / 2}$ for constructing unconditional confidence intervals

\begin{tabular}{|c|c|c|c|c|c|c|c|c|}
\hline$n$ & $\begin{array}{c}p=2 \\
\alpha=0.05\end{array}$ & $\alpha=0.10$ & $\begin{array}{c}p=3 \\
\alpha=0.05\end{array}$ & $\alpha=0.10$ & $\begin{array}{c}p=4 \\
\alpha=0.05\end{array}$ & $\alpha=0.10$ & $\begin{array}{c}p=5 \\
\alpha=0.05\end{array}$ & $\alpha=0.10$ \\
\hline 6 & 3.1900 & 2.4198 & 4.3705 & 3.1631 & 7.6337 & 5.0175 & - & - \\
\hline 7 & 2.8661 & 2.2340 & 3.5565 & 2.6903 & 4.9204 & 3.5130 & 8.5522 & 5.5248 \\
\hline 8 & 2.6894 & 2.1210 & 3.1478 & 2.4336 & 3.9086 & 2.9316 & 5.3637 & 3.8168 \\
\hline 9 & 2.5590 & 2.0442 & 2.8964 & 2.3029 & 3.3856 & 2.6167 & 4.2050 & 3.1612 \\
\hline 10 & 2.4664 & 1.9856 & 2.7330 & 2.1722 & 3.1018 & 2.4282 & 3.6301 & 2.7853 \\
\hline 11 & 2.3969 & 1.9402 & 2.6243 & 2.1005 & 2.9069 & 2.3003 & 3.2978 & 2.5723 \\
\hline 12 & 2.3539 & 1.9061 & 2.5375 & 2.0489 & 2.7699 & 2.2075 & 3.0582 & 2.4240 \\
\hline 13 & 2.3085 & 1.8863 & 2.4677 & 2.0041 & 2.6603 & 2.1428 & 2.8958 & 2.3143 \\
\hline 14 & 2.2762 & 1.8584 & 2.4163 & 1.9641 & 2.5822 & 2.0904 & 2.7835 & 2.2307 \\
\hline 15 & 2.2470 & 1.8430 & 2.3681 & 1.9327 & 2.5180 & 2.0388 & 2.6854 & 2.1731 \\
\hline 16 & 2.2291 & 1.8280 & 2.3356 & 1.9116 & 2.4651 & 2.0074 & 2.6121 & 2.1184 \\
\hline 17 & 2.2051 & 1.8124 & 2.3033 & 1.8897 & 2.4211 & 1.9749 & 2.5499 & 2.0772 \\
\hline 18 & 2.1922 & 1.8007 & 2.2836 & 1.8714 & 2.3850 & 1.9503 & 2.5011 & 2.0383 \\
\hline 19 & 2.1759 & 1.7964 & 2.2625 & 1.8602 & 2.3505 & 1.9265 & 2.4584 & 2.0115 \\
\hline 20 & 2.1647 & 1.7856 & 2.2418 & 1.8405 & 2.3222 & 1.9103 & 2.4234 & 1.9884 \\
\hline 21 & 2.1549 & 1.7749 & 2.2229 & 1.8342 & 2.3058 & 1.8951 & 2.3883 & 1.9641 \\
\hline 22 & 2.1434 & 1.7652 & 2.2074 & 1.8238 & 2.2758 & 1.8804 & 2.3664 & 1.9422 \\
\hline 23 & 2.1360 & 1.7644 & 2.1966 & 1.8102 & 2.2666 & 1.8657 & 2.3412 & 1.9243 \\
\hline 24 & 2.1240 & 1.7586 & 2.1866 & 1.8070 & 2.2502 & 1.8605 & 2.3173 & 1.9143 \\
\hline 25 & 2.1166 & 1.7471 & 2.1735 & 1.7963 & 2.2377 & 1.8388 & 2.2951 & 1.8973 \\
\hline 30 & 2.0857 & 1.7346 & 2.1301 & 1.7711 & 2.1749 & 1.8003 & 2.2252 & 1.8452 \\
\hline 35 & 2.0617 & 1.7151 & 2.1048 & 1.7482 & 2.1370 & 1.7791 & 2.1750 & 1.8127 \\
\hline 40 & 2.0525 & 1.7047 & 2.0809 & 1.7348 & 2.1148 & 1.7549 & 2.1429 & 1.7895 \\
\hline 45 & 2.0364 & 1.6993 & 2.0622 & 1.7217 & 2.0964 & 1.7467 & 2.1228 & 1.7674 \\
\hline 50 & 2.0347 & 1.6920 & 2.0597 & 1.7151 & 2.0775 & 1.7337 & 2.1034 & 1.7563 \\
\hline 60 & 2.0219 & 1.6868 & 2.0360 & 1.7037 & 2.0614 & 1.7176 & 2.0731 & 1.7341 \\
\hline 70 & 2.0076 & 1.6788 & 2.0226 & 1.6956 & 2.0390 & 1.7088 & 2.0590 & 1.7221 \\
\hline 80 & 2.0081 & 1.6765 & 2.0195 & 1.6889 & 2.0319 & 1.6966 & 2.0440 & 1.7096 \\
\hline 90 & 1.9979 & 1.6743 & 2.0095 & 1.6842 & 2.0210 & 1.6899 & 2.0352 & 1.7008 \\
\hline 100 & 1.9959 & 1.6676 & 2.0085 & 1.6825 & 2.0182 & 1.6872 & 2.0255 & 1.6991 \\
\hline 1000 & 1.9627 & 1.6512 & 1.9628 & 1.6478 & 1.9654 & 1.6494 & 1.9635 & 1.6490 \\
\hline
\end{tabular}

We numerically evaluated $E L_{2} / E L_{1}$ and $E L_{3} / E L_{1}$ and presented them in Table II. It is clear from the table values that $E L_{2}$ is in general either very close to $E L_{3}$ or smaller than $E L_{3}$, and the difference between them decreases as $n$ increases. Thus, we see that the conditional confidence interval is not only simple to construct but also narrower than the unconditional confidence interval. Furthermore, if $n-p$ is small and $\rho_{y . X}^{2}$ is small, then the usual $t$ interval is shorter than both conditional and unconditional intervals (see the values in Table II when $n=6, p=3$ and $n=6, p=5)$. Thus, the conditional combined method is preferable to the best of the $t$ procedures only when condition (20) holds and/or $n-p$ is moderately large. 
TABLE II Ratios of the Expected Lengths of $95 \%$ Confidence Intervals; $p=2$

\begin{tabular}{c|cc|cc|cc|cc|cc}
\hline \hline & $n=6$ & & $n=10$ & & $n=15$ & & $n=20$ & & $n=30$ & \\
$\rho_{y . X}^{2}$ & $\frac{E L_{2}}{E L_{1}}$ & $\frac{E L_{3}}{E L_{1}}$ & $\frac{E L_{2}}{E L_{1}}$ & $\frac{E L_{3}}{E L_{1}}$ & $\frac{E L_{2}}{E L_{1}}$ & $\frac{E L_{3}}{E L_{1}}$ & $\frac{E L_{2}}{E L_{1}}$ & $\frac{E L_{3}}{E L_{1}}$ & $\frac{E L_{2}}{E L_{1}}$ & $\frac{E L_{3}}{E L_{1}}$ \\
\hline 0.05 & 1.1770 & 1.2121 & 1.0538 & 1.0518 & 1.0188 & 1.0211 & 1.0052 & 1.0143 & 0.9935 & 0.9900 \\
0.10 & 1.1456 & 1.1510 & 1.0257 & 1.0157 & 0.9916 & 0.9867 & 0.9784 & 0.9657 & 0.9670 & 0.9655 \\
0.20 & 1.0801 & 1.1151 & 0.9671 & 0.9641 & 0.9349 & 0.9255 & 0.9224 & 0.9206 & 0.9117 & 0.9140 \\
0.30 & 1.0103 & 1.0275 & 0.9046 & 0.9113 & 0.8746 & 0.8719 & 0.8628 & 0.8652 & 0.8528 & 0.8542 \\
0.40 & 0.9354 & 0.9507 & 0.8375 & 0.8488 & 0.8097 & 0.8075 & 0.7988 & 0.8032 & 0.7895 & 0.7882 \\
0.50 & 0.8539 & 0.8773 & 0.7645 & 0.7630 & 0.7391 & 0.7367 & 0.7292 & 0.7299 & 0.7207 & 0.7176 \\
0.60 & 0.7637 & 0.7843 & 0.6838 & 0.6855 & 0.6611 & 0.6551 & 0.6522 & 0.6501 & 0.6447 & 0.6442 \\
0.70 & 0.6614 & 0.6741 & 0.5922 & 0.5931 & 0.5725 & 0.5733 & 0.5649 & 0.5633 & 0.5583 & 0.5610 \\
0.80 & 0.5400 & 0.5509 & 0.4835 & 0.4833 & 0.4675 & 0.4684 & 0.4612 & 0.4609 & 0.4558 & 0.4581 \\
0.90 & 0.3819 & 0.3863 & 0.3419 & 0.3410 & 0.3305 & 0.3310 & 0.3261 & 0.3279 & 0.3223 & 0.3217 \\
0.95 & 0.2700 & 0.2746 & 0.2418 & 0.2441 & 0.2337 & 0.2345 & 0.2306 & 0.2301 & 0.2279 & 0.2281 \\
\hline$\rho_{L}^{2}$ & 0.3142 & & 0.1446 & & 0.0848 & & 0.0597 & & 0.0375 & \\
\hline
\end{tabular}

TABLE II continued. $p=3$

\begin{tabular}{c|cc|cc|cc|cc|cc}
\hline \hline & $n=6$ & & $n=10$ & & $n=20$ & & $n=30$ & & $n=40$ & \\
$\rho_{y . X}^{2}$ & $\frac{E L_{2}}{E L_{1}}$ & $\frac{E L_{3}}{E L_{1}}$ & $\frac{E L_{2}}{E L_{1}}$ & $\frac{E L_{3}}{E L_{1}}$ & $\frac{E L_{2}}{E L_{1}}$ & $\frac{E L_{3}}{E L_{1}}$ & $\frac{E L_{2}}{E L_{1}}$ & $\frac{E L_{3}}{E L_{1}}$ & $\frac{E L_{2}}{E L_{1}}$ & $\frac{E L_{3}}{E L_{1}}$ \\
\hline 0.05 & 1.5578 & 1.6084 & 1.1552 & 1.1651 & 1.0695 & 1.0737 & 1.0387 & 1.0408 & 1.0134 & 1.0147 \\
0.10 & 1.5163 & 1.5652 & 1.1244 & 1.1423 & 1.0410 & 1.0461 & 1.0110 & 1.0132 & 0.9864 & 0.9884 \\
0.20 & 1.4296 & 1.4853 & 1.0601 & 1.0722 & 0.9814 & 0.9850 & 0.9532 & 0.9565 & 0.9300 & 0.9298 \\
0.30 & 1.3372 & 1.3845 & 0.9917 & 1.0051 & 0.9180 & 0.9205 & 0.8916 & 0.8957 & 0.8699 & 0.8715 \\
0.40 & 1.2380 & 1.2763 & 0.9181 & 0.9293 & 0.8499 & 0.8564 & 0.8255 & 0.8270 & 0.8054 & 0.8049 \\
0.50 & 1.1302 & 1.1726 & 0.8381 & 0.8480 & 0.7759 & 0.7793 & 0.7535 & 0.7550 & 0.7352 & 0.7356 \\
0.60 & 1.0108 & 1.0451 & 0.7496 & 0.7589 & 0.6940 & 0.6985 & 0.6740 & 0.6760 & 0.6576 & 0.6586 \\
0.70 & 0.8754 & 0.9035 & 0.6492 & 0.6562 & 0.6010 & 0.6045 & 0.5837 & 0.5859 & 0.5695 & 0.5687 \\
0.80 & 0.7148 & 0.7394 & 0.5301 & 0.5363 & 0.4907 & 0.4942 & 0.4766 & 0.4770 & 0.4650 & 0.4640 \\
0.90 & 0.5054 & 0.5256 & 0.3748 & 0.3809 & 0.3470 & 0.3490 & 0.3370 & 0.3378 & 0.3288 & 0.3291 \\
0.95 & 0.3574 & 0.3702 & 0.2650 & 0.2688 & 0.2454 & 0.2467 & 0.2383 & 0.2391 & 0.2325 & 0.2325 \\
\hline$\rho_{L}^{2}$ & 0.6100 & & 0.2882 & & 0.1694 & & 0.1194 & & 0.0749 & \\
\hline
\end{tabular}

Note: $\rho_{L}^{2}$ is the lower bound given in the right-hand side of (20);EL $<E L_{1}$ when (20) holds 
TABLE II continued. $p=5$

\begin{tabular}{c|cc|cc|cc|cc|cc}
\hline \hline & $n=6$ & & $n=10$ & & $n=20$ & & $n=30$ & & \multicolumn{2}{|c|}{$n=40$} \\
$\rho_{y \cdot X}^{2}$ & $\frac{E L_{2}}{E L_{1}}$ & $\frac{E L_{3}}{E L_{1}}$ & $\frac{E L_{2}}{E L_{1}}$ & $\frac{E L_{3}}{E L_{1}}$ & $\frac{E L_{2}}{E L_{1}}$ & $\frac{E L_{3}}{E L_{1}}$ & $\frac{E L_{2}}{E L_{1}}$ & $\frac{E L_{3}}{E L_{1}}$ & $\frac{E L_{2}}{E L_{1}}$ & $\frac{E L_{3}}{E L_{1}}$ \\
\hline 0.05 & 10.7729 & 10.3750 & 1.4860 & 1.5312 & 1.1171 & 1.1263 & 1.0571 & 1.0594 & 1.0326 & 1.0348 \\
0.10 & 10.4855 & 10.0999 & 1.4463 & 1.4949 & 1.0873 & 1.0912 & 1.0289 & 1.0328 & 1.0051 & 1.0078 \\
0.20 & 9.8859 & 9.5459 & 1.3636 & 1.4038 & 1.0251 & 1.0321 & 0.9701 & 0.9730 & 0.9476 & 0.9489 \\
0.30 & 9.2474 & 8.9105 & 1.2755 & 1.3155 & 0.9589 & 0.9638 & 0.9074 & 0.9100 & 0.8864 & 0.8886 \\
0.40 & 8.5614 & 8.2577 & 1.1809 & 1.2170 & 0.8878 & 0.8960 & 0.8401 & 0.8425 & 0.8207 & 0.8211 \\
0.50 & 7.8155 & 7.5403 & 1.0780 & 1.1085 & 0.8104 & 0.8146 & 0.7669 & 0.7671 & 0.7492 & 0.7505 \\
0.60 & 6.9904 & 6.6793 & 0.9642 & 0.9894 & 0.7249 & 0.7289 & 0.6859 & 0.6863 & 0.6701 & 0.6729 \\
0.70 & 6.0538 & 5.8581 & 0.8350 & 0.8609 & 0.6278 & 0.6333 & 0.5940 & 0.5940 & 0.5803 & 0.5815 \\
0.80 & 4.9429 & 4.7997 & 0.6818 & 0.7039 & 0.5126 & 0.5147 & 0.4850 & 0.4858 & 0.4738 & 0.4736 \\
0.90 & 3.4952 & 3.3807 & 0.4821 & 0.4977 & 0.3624 & 0.3651 & 0.3430 & 0.3437 & 0.3350 & 0.3342 \\
0.95 & 2.4715 & 2.3903 & 0.3409 & 0.3505 & 0.2563 & 0.2578 & 0.2425 & 0.2433 & 0.2369 & 0.2376 \\
\hline$\rho_{L}^{2}$ & 0.9918 & & 0.5698 & & 0.2387 & & 0.1499 & & 0.1091 & \\
\hline
\end{tabular}

Note: $\rho_{L}^{2}$ is the lower bound given in the right-hand side of (20); $E L_{2}<E L_{1}$ when (20) holds

\section{POWER FUNCTION}

We observed in the preceding section that the conditional method performs better than the unconditional method, and hence we consider only the power function of the conditional test based on (12). Consider the hypotheses

$$
H_{0}: \mu \leq \mu_{0} \text { vs. } H_{a}: \mu>\mu_{0} .
$$

The conditional non-null distribution (given $T^{2}$ ) is the noncentral $t$ distribution with $\mathrm{df}=$ $n-p-1$ and the noncentrality parameter

$$
\delta(Q)=\frac{\sqrt{n}\left(\mu-\mu_{0}\right)}{\sqrt{\sigma_{y y \cdot X}} \sqrt{1+Q}},
$$

where $\mu$ is the true value and $\mu_{0}$ is the specified value of the mean. The unconditional power of a right-tail test can be expressed as

$$
E_{Q}\left[P\left(t_{n-p-1}(\delta(Q))>t_{n-p-1,1-\alpha}\right)\right] .
$$

Again, using the fact that $1+Q$ is distributed as the reciprocal of a beta $((n-p) / 2, p / 2)$ random variable, the power can be computed using the numerical integration

$$
1-\frac{\Gamma(n / 2)}{\Gamma(p / 2) \Gamma((n-p) / 2)} \int_{0}^{1} G\left(c_{1} ; n-p-1, \delta\left(u^{-1}\right)\right) u^{p / 2-1}(1-u)^{(n-p) / 2-1} d u,
$$

where $c_{1}=t_{n-p-1,1-\alpha}$ and $G(x ; m, d)$ denotes the cdf of a noncentral $t$ random variable with $\mathrm{df}=m$ and the noncentrality parameter $d$.

Although it is not difficult to compute (20), a simple approximate power expression can be obtained from (19), and is given by

$$
P\left(t_{n-p-1}\left(\delta(E(Q))>t_{n-p-1,1-\alpha}\right) .\right.
$$


Noting that $E(Q)=(n-2) /(n-p-2)$, for a given level of significance $\alpha, p$ and $\eta=$ $\sqrt{\left(\mu-\mu_{0}\right) / \sigma_{y y \cdot X}}$, an approximate sample size $n$ that is required to attain a power of $1-\beta$ satisfies

$$
P\left(t_{n-p-1}\left(\delta_{1}\right)>t_{n-p-1,1-\alpha}\right)=1-\beta
$$

where

$$
\delta_{1}=\frac{\sqrt{n}\left(\mu-\mu_{0}\right)}{\sqrt{\sigma_{y y \cdot X}}}\left(\frac{n-p-2}{n-2}\right)^{\frac{1}{2}} .
$$

In order to understand the validity of the approximation, we computed the exact power using (24) and the approximate power based on (26) for various values of $n, \eta=\sqrt{\left(\mu-\mu_{0}\right) / \sigma_{y y . X}}$ and $p=2,3$ and 4 . These powers are presented TABLE II. We see from the table values that the approximate powers are close to the exact powers provided $n$ is moderately large in comparison to $p$. Our extensive numerical studies for various values of $p$ (not reported here) showed that the approximation is very satisfactory for values of $n \geq 5 p$. An advantage of this approximation is that it only involves the computation of the noncentral cdf with fixed noncentrality parameter (when $\eta, n$ and $p$ are given), and so the power computation can be carried out using freely available PC calculators such as StatCalc (http://www.etext.net/catalog/StatCalc/) or online calculator available at http://calculators.stat.ucla.edu/.

TABLE III Exact powers (24) and approximate powers (25) of the conditional test when $\alpha=0.05$

$p=2$

\begin{tabular}{c|cc|cc|cc|cc|cc}
\hline \hline & $n=6$ & & $n=8$ & & $n=12$ & & $n=16$ & & $n=20$ & \\
$\eta=\frac{\mu-\mu_{0}}{\sqrt{\sigma_{y y \cdot X}}}$ & Exact & Appr. & Exact & Appr. & Exact & Appr. & Exact & Appr. & Exact & Appr. \\
\hline 0.00 & 0.050 & 0.050 & 0.050 & 0.050 & 0.050 & 0.050 & 0.050 & 0.050 & 0.050 & 0.050 \\
0.10 & 0.079 & 0.080 & 0.085 & 0.086 & 0.091 & 0.091 & 0.096 & 0.096 & 0.101 & 0.101 \\
0.20 & 0.120 & 0.121 & 0.137 & 0.137 & 0.153 & 0.153 & 0.168 & 0.168 & 0.182 & 0.182 \\
0.30 & 0.174 & 0.175 & 0.206 & 0.207 & 0.237 & 0.237 & 0.266 & 0.267 & 0.295 & 0.295 \\
0.40 & 0.239 & 0.242 & 0.291 & 0.293 & 0.341 & 0.342 & 0.387 & 0.388 & 0.431 & 0.432 \\
0.50 & 0.314 & 0.321 & 0.389 & 0.393 & 0.458 & 0.461 & 0.520 & 0.522 & 0.576 & 0.578 \\
0.60 & 0.396 & 0.407 & 0.494 & 0.501 & 0.577 & 0.583 & 0.649 & 0.653 & 0.711 & 0.714 \\
0.70 & 0.480 & 0.498 & 0.597 & 0.607 & 0.689 & 0.697 & 0.763 & 0.768 & 0.820 & 0.824 \\
0.80 & 0.565 & 0.588 & 0.693 & 0.706 & 0.785 & 0.794 & 0.852 & 0.858 & 0.899 & 0.903 \\
0.90 & 0.645 & 0.673 & 0.776 & 0.792 & 0.861 & 0.870 & 0.915 & 0.920 & 0.949 & 0.952 \\
1.00 & 0.718 & 0.750 & 0.844 & 0.860 & 0.916 & 0.924 & 0.955 & 0.959 & 0.977 & 0.979 \\
\hline
\end{tabular}


TABLE III continued.

$p=3$

\begin{tabular}{|c|c|c|c|c|c|c|c|c|c|c|}
\hline$\eta=\frac{\mu-\mu_{0}}{\sqrt{\sigma_{y y . X}}}$ & $\begin{array}{l}n=8 \\
\text { Exact }\end{array}$ & Appr. & $\begin{array}{c}n=12 \\
\text { Exact }\end{array}$ & Appr. & $\begin{array}{c}n=16 \\
\text { Exact }\end{array}$ & Appr. & $\begin{array}{c}n=20 \\
\text { Exact }\end{array}$ & Appr. & $\begin{array}{c}n=24 \\
\text { Exact }\end{array}$ & Appr. \\
\hline 0.00 & 0.050 & 0.050 & 0.050 & 0.050 & 0.050 & 0.050 & 0.050 & 0.050 & 0.050 & 0.050 \\
\hline 0.10 & 0.084 & 0.086 & 0.089 & 0.090 & 0.099 & 0.099 & 0.108 & 0.108 & 0.117 & 0.117 \\
\hline 0.20 & 0.133 & 0.137 & 0.148 & 0.149 & 0.176 & 0.177 & 0.204 & 0.205 & 0.231 & 0.232 \\
\hline 0.30 & 0.196 & 0.205 & 0.226 & 0.229 & 0.283 & 0.284 & 0.338 & 0.339 & 0.390 & 0.390 \\
\hline 0.40 & 0.271 & 0.288 & 0.322 & 0.328 & 0.413 & 0.416 & 0.495 & 0.497 & 0.568 & 0.570 \\
\hline 0.50 & 0.353 & 0.383 & 0.428 & 0.441 & 0.551 & 0.557 & 0.651 & 0.656 & 0.733 & 0.736 \\
\hline 0.60 & 0.439 & 0.484 & 0.536 & 0.558 & 0.681 & 0.691 & 0.785 & 0.791 & 0.857 & 0.861 \\
\hline 0.70 & 0.524 & 0.585 & 0.640 & 0.669 & 0.791 & 0.804 & 0.882 & 0.888 & 0.935 & 0.938 \\
\hline 0.80 & 0.605 & 0.679 & 0.732 & 0.767 & 0.874 & 0.887 & 0.942 & 0.948 & 0.974 & 0.977 \\
\hline 0.90 & 0.679 & 0.762 & 0.809 & 0.846 & 0.930 & 0.941 & 0.975 & 0.979 & 0.992 & 0.993 \\
\hline 1.00 & 0.745 & 0.831 & 0.870 & 0.905 & 0.964 & 0.972 & 0.991 & 0.993 & 0.998 & 0.998 \\
\hline
\end{tabular}

TABLE III continued.

$p=4$

\begin{tabular}{c|cc|cc|cc|cc|cc}
\hline \hline & $n=8$ & & $n=12$ & & $n=16$ & & $n=20$ & & $n=24$ & \\
$\eta=\frac{\mu-\mu_{0}}{\sqrt{\sigma_{y y . X}}}$ & Exact & Appr. & Exact & Appr. & Exact & Appr. & Exact & Appr. & Exact & Appr. \\
\hline 0.00 & 0.050 & 0.050 & 0.050 & 0.050 & 0.050 & 0.050 & 0.050 & 0.050 & 0.050 & 0.050 \\
0.10 & 0.094 & 0.096 & 0.103 & 0.103 & 0.111 & 0.112 & 0.120 & 0.120 & 0.128 & 0.128 \\
0.20 & 0.162 & 0.165 & 0.187 & 0.188 & 0.214 & 0.214 & 0.240 & 0.240 & 0.265 & 0.266 \\
0.30 & 0.252 & 0.260 & 0.304 & 0.306 & 0.355 & 0.357 & 0.405 & 0.406 & 0.452 & 0.453 \\
0.40 & 0.358 & 0.377 & 0.442 & 0.448 & 0.519 & 0.523 & 0.588 & 0.591 & 0.650 & 0.652 \\
0.50 & 0.470 & 0.504 & 0.585 & 0.597 & 0.677 & 0.685 & 0.752 & 0.758 & 0.812 & 0.816 \\
0.60 & 0.580 & 0.630 & 0.714 & 0.733 & 0.807 & 0.817 & 0.872 & 0.878 & 0.917 & 0.920 \\
0.70 & 0.680 & 0.743 & 0.819 & 0.841 & 0.898 & 0.908 & 0.944 & 0.949 & 0.970 & 0.972 \\
0.80 & 0.766 & 0.833 & 0.894 & 0.915 & 0.952 & 0.960 & 0.979 & 0.982 & 0.991 & 0.992 \\
0.90 & 0.835 & 0.900 & 0.943 & 0.959 & 0.980 & 0.985 & 0.993 & 0.995 & 0.998 & 0.998 \\
1.00 & 0.888 & 0.944 & 0.972 & 0.983 & 0.993 & 0.995 & 0.998 & 0.999 & 1.000 & 1.000 \\
\hline
\end{tabular}

\section{AN EXAMPLE}

To illustrate the methods of this paper, we generated a sample of 20 observations from $N(\mu, \Sigma)$, where

$$
\mu=\left(\begin{array}{l}
4 \\
4 \\
4
\end{array}\right) \text { and } \Sigma=\left(\begin{array}{ccc}
3 & 0.9798 & 2.0785 \\
0.9789 & 2 & 1.9799 \\
2.0785 & 1.9799 & 4
\end{array}\right)
$$

The data points are given in Table IV. The summary statistics are

$\left(\bar{y}, \bar{x}_{1}, \bar{x}_{2}\right)=(3.9064,4.1602,4.2912), \quad\left(\begin{array}{cc}w_{y y} & W_{y y . X} \\ W_{y y . X}^{\prime} & W_{X X}\end{array}\right)=\left(\begin{array}{ccc}57.9621 & 24.7029 & 22.7379 \\ - & 28.4999 & 11.6107 \\ - & - & 55.7325\end{array}\right)$, 
$W_{X X}^{-1}=\left(\begin{array}{cc}3.83421 E-2 & -7.98701 E-3 \\ - & 1.960696 E-2\end{array}\right), \hat{\mu}=4.0953, \hat{\sigma}_{y y . X}=1.9648$ and $\rho_{y \cdot X}^{2}=0.4238$.

The standard deviations are $s_{u_{1}}=1.747, s_{u_{2}}=1.397$ and $s_{u_{3}}=1.895$. The critical points $t_{19,0.975}=2.0930$ and $T_{u, 0.975}=2.2418$. Using these statistics, we computed the following confidence intervals for $\mu$.

TABLE IV Simulated data; $n=20, p=3$

\begin{tabular}{c|ccc|ccc}
\hline \hline & $u_{1}$ & $u_{2}$ & $u_{3}$ & $y=u_{1}$ & $x_{1}=u_{1}-u_{2}$ & $x_{2}=u_{1}-u 3$ \\
\hline 1 & 0.9913 & 2.3451 & 2.4441 & 0.9913 & -1.3538 & -1.4528 \\
2 & 1.8680 & 3.5647 & 1.1850 & 1.8680 & -1.6967 & 0.6830 \\
3 & 4.5551 & 4.7550 & 5.2841 & 4.5551 & -0.1999 & -0.7290 \\
4 & 4.0409 & 2.9949 & 6.6683 & 4.0409 & 1.0460 & -2.6274 \\
5 & 5.0872 & 5.3200 & 5.1576 & 5.0872 & -0.2328 & -0.0704 \\
6 & 3.4412 & 5.6166 & 3.1288 & 3.4412 & -2.1754 & 0.3124 \\
7 & 6.1288 & 5.5200 & 7.6320 & 6.1288 & 0.6088 & -1.5032 \\
8 & 5.7505 & 4.4716 & 2.6449 & 5.7505 & 1.2789 & 3.1056 \\
9 & 4.9089 & 3.7319 & 6.7311 & 4.9089 & 1.1770 & -1.8222 \\
10 & 3.4542 & 2.9163 & 2.1313 & 3.4542 & 0.5379 & 1.3229 \\
11 & 5.4352 & 3.6849 & 3.7720 & 5.4352 & 1.7503 & 1.6632 \\
12 & 3.7283 & 5.6323 & 4.5298 & 3.7283 & -1.9040 & -0.8015 \\
13 & 4.8681 & 4.7071 & 2.5703 & 4.8681 & 0.1610 & 2.2978 \\
14 & 5.0751 & 4.4576 & 4.2097 & 5.0751 & 0.6175 & 0.8654 \\
15 & 2.4324 & 2.1372 & 1.0812 & 2.4324 & 0.2952 & 1.3512 \\
16 & 4.8654 & 4.1743 & 4.5202 & 4.8654 & 0.6911 & 0.3452 \\
17 & 9.1629 & 7.4908 & 5.5794 & 9.1629 & 1.6721 & 3.5835 \\
18 & 3.7241 & 4.3609 & 1.7385 & 3.7241 & -0.6368 & 1.9856 \\
19 & 3.4018 & 1.6481 & 2.5608 & 3.4018 & 1.7537 & 0.8410 \\
20 & 2.9055 & 3.6752 & 4.5584 & 2.9055 & -0.7697 & -1.6529 \\
\hline
\end{tabular}

The $95 \%$ t-intervals

(a) $\bar{u}_{1} \pm t_{19,0.975} \frac{s_{u_{1}}}{\sqrt{n}}=4.2912 \pm 0.8174 ; \hat{\rho}_{u_{1} .\left(u_{1}-u_{2}, u_{1}-u_{3}\right)}^{2}=0.4238$

(b) $\bar{u}_{2} \pm t_{19,0.975} \frac{s_{u_{2}}}{\sqrt{n}}=4.1602 \pm 0.6536 ; \hat{\rho}_{u_{2} .\left(u_{2}-u_{1}, u_{2}-u_{3}\right)}^{2}=0.09864$

(c) $\bar{u}_{3} \pm t_{19,0.975} \frac{s_{u_{3}}}{\sqrt{n}}=3.9064 \pm 0.8868 ; \hat{\rho}_{u_{3} .\left(u_{3}-u_{2}, u_{3}-u_{1}\right)}^{2}=0.5104$

The conditional interval in $(13)=4.0953 \pm 0.6793$

The unconditional interval in $(17)=4.0953 \pm 0.7026$

The interval (b) is the shortest among all the intervals. We also note that, for the conditional interval to be the shortest, we must have

$$
\min \left\{\rho_{u_{1} \cdot\left(u_{1}-u_{2}\right),\left(u_{1}-u_{2}\right)}^{2}, \rho_{u_{1} \cdot\left(u_{1}-u_{2}\right),\left(u_{1}-u_{2}\right)}^{2}, \rho_{u_{1} \cdot\left(u_{1}-u_{2}\right),\left(u_{1}-u_{2}\right)}^{2}\right\}>0.1194 .
$$


(see Table II, $n=20, p=3$ ). Since the minimum of the sample squared multiple correlation coefficients is 0.09864 , we do not have any evidence in favor of (28). Therefore, as already observed, the conditional approach did not produce the shortest interval. We also see that, among all the point estimates, the MLE is very close to the true mean 4.

\section{CONCLUDING REMARKS}

We observed from the preceding sections that the unconditional approach is not only simple to use but also better than the unconditional method for constructing confidence interval for the common mean $\mu$. Furthermore, if the sample size is sufficiently large, then the conditional approach may yield better results than the ones based on the individual $t$ procedures. For a fixed $p$ and $\alpha=0.05$, we computed the least value of $n$ for which $\rho_{L}^{2}=1-\left(\frac{t_{n-1,1-\alpha / 2}}{t_{n-p, 1-\alpha / 2}}\right)^{2}\left(\frac{n-p}{n-1}\right)>0.05$. Based on a linear fit of these pairs of $(n, p)$, we found that $\rho_{L}^{2}>0.05$ for any $n>20 p-15$. This implies that $n$ must be at least $20 p-15$ for the conditional approach offers improvement over the best of the $t$ procedures for any $\rho_{y . X}^{2}>0.05$. For moderate sample sizes, to check if the conditional combined approach is superior to the best of individual $t$ methods, one should test whether the minimum of the squared sample multiple correlation coefficients is greater than $\rho_{L}^{2}=1-\left(\frac{t_{n-1,1-\alpha / 2}}{t_{n-p, 1-\alpha / 2}}\right)^{2}\left(\frac{n-p}{n-1}\right)$. It is difficult to obtain an exact test to verify this condition. Therefore, in practice one may want to compute all the $t$ intervals based on the individual components and the conditional confidence interval, and then choose the shortest of the intervals for applications.

\section{Acknowledgement}

The authors are thankful to a referee for reviewing this article. 


\section{References}

Berry, C. J. (1987) Equivariant estimation of a normal mean using a normal concomitant variable for covariance adjustment. The Canadian Journal of Statistics, 15, 177-183.

Cohen, A. and Sackrowitz, H. B. (1984) Testing hypotheses about the common mean of normal distributions. Journal of Statistical Planning and Inference, 9, 207-227.

Halperin, M. (1961) Almost linearly-optimum combination of unbiased estimates. Journal of the American Statistical Association, 56, 36-43

Jin, C. and Berry, C. J. (1993) Equivariant estimation of a normal mean vector using a normal concomitant vector for covariance adjustment. Communications in Statistics - Theory and Methods, 22, 335-346.

Krishnamoorthy, K. and Rohatgi, V. K. (1990). Unbiased estimation of the common mean of a multivariate normal distribution. Communication Statistics-Theory Methods, 19, $1803-1812$

Krishnamoorthy, K. and Lu, Y. (2003) Inferences on the common mean of several normal populations based on the generalized variable method. Biometrics, 59, 237-247

Muirhead, R. J. (1982) Aspects of Multivariate Statistical Theory, Wiley, New York.

Tan, M. and Gleser, L. J. (1993) Improved point and confidence interval estimators of mean response in simulation when control variates are used, Communications in Statistics Simulation and Computation, 22, 1211-1220.

Yu, P. L. H., Sun, Y. and Sinha, B. K. (1999) On exact confidence intervals for the common mean of several normal populations. Journal of Statistical Planning and Inference, 81, $263-277$.

Zhou, L. and Mathew, T. (1993) Combining independent tests in linear models. Journal of the American Statistical Association, 88, 650-655. 